\title{
Modelagem Matemática do Aedes aegypti com a utilização de mecanismos de combate à Dengue
}

\author{
Luísa Lopes Ferreira Gomes* Roberto C. A. Thomé \\ DIPPG - Diretoria de Pesquisa e Pós Graduação, \\ CEFET/RJ - Centro de Educação Tecnológica Celso Suckow da Fonseca, \\ 20271-110, Maracanã, Rio de Janeiro, RJ \\ E-mail: luisalopesfg@gmail.com, rthome@cefet-rj.br
}

\section{RESUMO}

O Aedes aegypti é o mosquito transmissor da Dengue e também da Febre Amarela. É um inseto cosmopolita, encontrado principalmente em países de clima tropical, como afirmado em [2]. O mosquito macho alimenta-se exclusivamente de frutas. A fêmea, no entanto, necessita de sangue para o amadurecimento dos ovos que são depositados separadamente nas paredes internas de objetos, próximos a extensas superfícies de água limpa, local que lhes oferece melhores condições de sobrevivência. Geralmente os sintomas da Dengue Clássica são febre alta, dores de cabeça, nas costas e na região atrás dos olhos, porém quando o ser humano é infectado pela segunda vez pelo Aedes aegypti, a chamada Dengue Hemorrágica, apresenta sintomas como hemorragia em vários órgãos e choque circulatório podendo levar o paciente ao óbito.

Diante da realidade da doença no país, apresentamos um modelo de controle ótimo de mosquitos, baseado na análise do efeito da introdução de insetos machos estéreis no meio ambiente, baseado no modelo proposto por Esteva \& Young em [1]. Em [1] foi estudada a aplicação do método de liberação de mosquitos Aedes aegypti, conhecida como técnica SIT (Sterile Insect Technique). Essa técnica tem-se mostrado muito eficiente no controle de pragas agrícolas e aparece como uma medida alternativa a técnica usual de aplicação de inseticidas, que, além de promover resistência do inseto ao produto químico utilizado, é não seletiva, isto é, prejudica outras espécies que vivem no mesmo habitat do mosquito.

O ciclo dos mosquitos é dividido em dois estágios: fase aquática (ovos, larvas e pupas) e fase alada (mosquitos adultos). Nosso modelo considera esses dois estágios e é baseado em equações diferenciais ordinárias que descrevem a dinâmica dos mosquitos na fase aquática, dos mosquitos fêmeas imaturas (antes de acasalar), dos mosquitos fêmeas fertilizadas (depois de acasalar), dos mosquitos fêmeas não-fertilizadas (depois de acasalar), dos mosquitos machos (macho natural) e dos mosquitos machos estéreis (irradiados).

Utilizaremos a notação $\mathrm{A}(\mathrm{t})$ para população de mosquitos na fase aquática, $\mathrm{I}(\mathrm{t})$ para população de mosquitos fêmeas imaturas (antes de acasalar), $\mathrm{F}(\mathrm{t})$ para população de mosquitos fêmeas fertilizadas (após acasalar), $\mathrm{U}(\mathrm{t})$ para população de mosquitos fêmeas não-fertilizadas (depois de acasalar), $\mathrm{M}(\mathrm{t})$ para população de mosquitos machos naturais e $\mathrm{S}(\mathrm{t})$ para população de mosquitos machos estéreis. As taxas de mortalidade per capita dos mosquitos nas diferentes fases serão representadas por $\mu$ seguido da respectiva variável de estado. A taxa $\phi(1-\mathrm{A} / \mathrm{C})$ representa a oviposição da fêmea fertilizada, onde $\phi$ é a taxa de oviposição intrínseca e $\mathrm{C}$ é a capacidade do meio relacionada ao número de nutrientes, espaço, etc. A taxa per capita $\gamma$ representa a mudança da fase aquática para a fase alada, onde uma proporção $r$ são de fềmeas e (1-r) são de machos. A taxa de probabilidade de encontro dos mosquitos fêmeas com machos naturais é dada por $\beta \mathrm{M} /(\mathrm{M}+\mathrm{S})$ e com machos estéreis é dada por $\beta_{\mathrm{s}} \mathrm{M} /(\mathrm{M}+\mathrm{S})$, onde $\beta$ é a 
taxa de acasalamento de mosquitos naturais e $\beta_{\mathrm{s}}$ é a taxa de acasalamento de mosquitos estéreis. Assumiremos que a população de mosquitos estéreis $\mathrm{S}$ são colocados no meio ambiente a uma taxa constante $\alpha$. A dinâmica do controle biológico é descrita da seguinte maneira:

$$
\left\{\begin{array}{l}
\frac{d A}{d t}=\phi\left(1-\frac{A}{C}\right) F-\left(\gamma+\mu_{A}\right) A \\
\frac{d I}{d t}=r \gamma A-\frac{\beta M I}{M+S}-\frac{\beta_{S} S I}{M+S}-\mu_{I} I \\
\frac{d F}{d t}=\frac{\beta M I}{M+S}-\mu_{F} F \\
\frac{d U}{d t}=\frac{\beta S S I}{M+S}-\mu_{U} U \\
\frac{d M}{d t}=(1-r) \gamma A-\mu_{M} M \\
\frac{d S}{d t}=\alpha-\mu_{S} S .
\end{array}\right.
$$

A partir dessa dinâmica, estudaremos os pontos de equilíbrio (trivial e nãotriviais), além de analisar a estabilidade do sistema dinâmico (ver [3]). Podemos observar que a variável de estado U (População de Mosquitos Fêmeas Não-Fertilizadas) do problema está desacoplada do sistema dinâmico dado em (1), isto é, as outras equações não dependem da variável U. Ao contrário do proposto por Esteva \& Young, optamos por manter a variável U no problema para analisar o comportamento de cada variável de estado em conjunto e de todas as etapas que ocorrem no sistema. Realizaremos simulações numéricas utilizando o MatLab com dados extraídos da literatura moderna e variações em parâmetros para obtenção de melhores resultados.

Além da análise do mecanismo de combate à dengue exposto (controle biológico), realizaremos comparações e combinações entre diversos mecanismos de controle como larvicida, adulticida, controle mecânico entre outros, bem como estratégias de utilização desses métodos em conjunto, levando em conta fatores como com o propósito de identificar a melhor estratégia no combate ao Aedes aegypti.

Palavras-chave: Dengue, Aedes aegypti, Técnica de Insetos Estéreis, Modelagem Matemática.

\section{Referências}

[1] Esteva L. \& Yang H. M.: Mathematical model to assess the control of Aedes aegypti mosquitoes by the sterile insect technique. Mathematical Biosciences 198, 2005.

[2] Tauil, P.L.: Aspectos críticos do controle do dengue no Brasil. Cad. Saúde Pública, 18: 867-871 (2002).

[3] Thomé R.C.A., Yang H. M. \& Esteva L.: Optimal control of Aedes aegypti mosquitoes by the sterile insect technique and insecticide. Mathematical Biosciences 223, 2010. 